HIFAN 1696

Approximate analytical solutions for continuously focused beams and single-species plasmas in thermal equilibrium

Edward A. Startsev1 and Steven M. Lund2

1Princeton Plasma Physics Laboratory, Princeton, New Jersey 08543, USA

2Lawrence Livermore National Laboratory, Livermore, California 94550, USA

Accelerator Fusion Research Division

Ernest Orlando Lawrence Berkeley National Laboratory

University of California

Berkeley, California 94720

\title{
December 2008
}

This work was supported by the Director, Office of Science, Office of Fusion Energy Sciences, of the U.S. Department of Energy under Contract No. DE-AC02-05CH11231. 


\title{
Approximate analytical solutions for continuously focused beams and single-species plasmas in thermal equilibrium
}

\author{
Edward A. Startsev ${ }^{1, a)}$ and Steven M. Lund ${ }^{2, b)}$ \\ ${ }_{1}^{1}$ Princeton Plasma Physics Laboratory, Princeton, New Jersey 08543, USA \\ ${ }^{2}$ Lawrence Livermore National Laboratory, Livermore, California 94550. USA
}

\begin{abstract}
Simple, two-dimensional Vlasov-Poisson descriptions of thermal equilibrium have been applied to both an unbunched ion beam propagating in a continuous linear focusing channel and an unneutralized, single-species plasma confined in a Penning-Malmberg trap geometry. In scaled variables, these two thermal equilibrium systems result in an identical nonlinear equation that must be solved to describe the radial density and/or potential of the equilibrium. Numerous publications have been based on numerical solutions of the highly nonlinear equilibrium equation. Following a brief review of the thermal equilibrium model, we construct approximate, closed-form analytical solutions to the equilibrium equation that are accurate over a wide range of system parameters.
\end{abstract}

\section{INTRODUCTION}

The concept of thermal equilibrium has proven useful in a wide range studies of continuous focusing models of accelerators $^{1-3}$ and pure electron plasmas confined in Penning-Malmberg traps. ${ }^{1,4}$ Within a Vlasov-Poisson model, concavity arguments show that the thermal equilibrium distributions are stable to both small- and large-amplitude perturbations. ${ }^{5-7}$ Although beam and plasma distributions are not typically expected to be produced in thermal equilibrium form, thermal equilibrium represents the state that collisions will tend to drive the system towards. In this context, thermal equilibrium is the ideal, stable state that the system must ultimately relax to. Thus, it is natural to express properties of realistic systems in terms of deviations from the thermal equilibrium state.

Thermal equilibrium descriptions of beam and nonneutral plasma systems can be solved analytically when the space-charge strength is sufficiently small. However, when space-charge becomes strong, Debye screening results in a flat-density core out to the finite characteristic radial edge of the beam or plasma where the density rapidly drops to exponentially small values. ${ }^{1,3,4,8-10}$ This results in a highly nonlinear ordinary differential equation to describe the radial density or potential profile of the system. Properties of the equilibrium solution in this regime have previously been studied numerically or in limiting form, ${ }^{1-4,11-15}$ or with a poorly convergent series expansion. ${ }^{16,17}$

Given the intrinsic interest of the thermal equilibrium systems, it is desirable to develop analytical models to describe the equilibrium profile in regimes of strong spacecharge strength. Here, we construct an approximate, closedform equilibrium solution that is valid over a wide parameter range corresponding to strong space-charge strength. This solution is potentially useful to describe both beam and non-neutral plasma systems associated with a variety of ex-

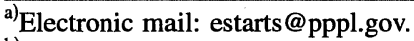

${ }^{b}$ Electronic mail: smlund@llnl.gov.
}

perimental devices. The relatively simple form of the solutions can help improve understanding of the thermal equilibrium properties of these systems and enable analytical progress on problems that were previously accessible only numerically.

\section{THERMAL EQUILIBRIUM MODEL}

Here we review simple Vlasov-Poisson model descriptions of thermal equilibrium systems corresponding to a charged particle beam in a continuous focusing channel (Sec. II A) and a single-species plasma confined in a PenningMalmberg trap geometry (Sec. II B). It is shown (Sec. II C) that both these formulations lead to identical equations in scaled variables that describe the radial potential and density profile of the equilibria. Properties of the equilibrium solution are parametrically summarized using numerical methods (Sec. II D).

\section{A. Beam in a continuous focusing channel}

Descriptions of correspondences between charged particle beams propagating in realistic, periodic focusing lattices and beams in continuous focusing lattices can be found in the literature. ${ }^{1-3,18}$ The continuous focusing model can be directly applied to periodic quadrupole focusing lattices using these correspondences, and to periodic solenoidal focusing lattices if the results are interpreted in a rotating "Larmor" frame. ${ }^{1,18}$ The continuous model is expected to more accurately represent stable beams in periodic lattices when the applied focusing strength is relatively weak. ${ }^{3,19,20}$

The continuous-focusing model of charged particle beams with a thermal equilibrium distribution has been extensively studied by Reiser ${ }^{2}$ and Davidson. ${ }^{1,3}$ A detailed review of the model can also be found in Ref. 17. Although the model can only be regarded as a highly idealized representation of a real beam evolving in a periodic focusing lattice, the model is nevertheless useful to illustrate basic physics 
and scaling properties since it is a smooth distribution with reasonable limits in both low- and high-intensity spacecharge regimes.

Consider an infinitely long, unbunched $(\partial / \partial z=0)$ beam of ions of charge $q$ and rest mass $m$. All particles propagate with axial velocity $\beta_{b} c=$ const. Here, $c$ is the speed of light in vacuo. The beam phase-space is described in terms of the transverse spatial coordinates $\mathbf{x}_{\perp}$ of the particles and the angles $\mathbf{x}_{\perp}^{\prime}$ that the particles make with the axis of the system. The single-particle Hamiltonian

$$
H_{\perp}=\frac{1}{2} \mathbf{x}_{\perp}^{\prime 2}+\frac{1}{2} k_{\beta 0}^{2} \mathbf{x}_{\perp}^{2}+\frac{q}{m \gamma_{b}^{3} \beta_{b}^{2} c^{2}} \phi,
$$

is a constant of the motion. Here, $k_{\beta 0}^{2}=$ const $>0$ is the continuous, applied focusing force of the channel, $\gamma_{b}=1 / \sqrt{1-\beta_{b}^{2}}=$ const is the axial relativistic gamma factor, and $\phi$ is the self-field potential generated by the beam spacecharge. Thermal equilibrium is specified by the distribution

$$
f\left(\mathbf{x}_{\perp}, \mathbf{x}_{\perp}^{\prime}\right)=\frac{m \gamma_{b} \beta_{b}^{2} c^{2} \hat{n}}{2 \pi T} \exp \left(-\frac{m \gamma_{b} \beta_{b}^{2} c^{2} H_{\perp}}{T}\right) .
$$

Here, $T=$ const is the thermodynamic temperature (expressed in energy units) and $\hat{n}=$ const is a characteristic density scale. The beam density can be calculated from Eq. (2) as

$$
n=\int d^{2} x_{\perp}^{\prime} f_{\perp}=\hat{n} \exp \left[-\left(\frac{m \gamma_{b} \beta_{b}^{2} c^{2} k_{\beta 0}^{2}}{2 T} r^{2}+\frac{q}{\gamma_{b}^{2} T} \phi\right)\right] .
$$

The proof in Ref. 21 shows that the solution to the twodimensional Poisson equation $\nabla_{\perp}^{2} \phi=-4 \pi q n$ in radially unbounded geometry is axisymmetric $(\partial / \partial \theta=0)$ with

$$
\frac{1}{r} \frac{\partial}{\partial r} r \frac{\partial}{\partial r} \phi=-4 \pi q \hat{n} \exp \left[-\left(\frac{m \gamma_{b} \beta_{b}^{2} c^{2} k_{\beta 0}^{2}}{2 T} r^{2}+\frac{q}{\gamma_{b}^{2} T} \phi\right)\right] \text {. }
$$

Here, $r=\left|\mathbf{x}_{\perp}\right|$ is the radial coordinate. Without loss of generality, we take a potential reference choice of $\phi(r=0)=0$ and consistently identify the on-axis density of the beam as $n(r=0)=\hat{n}$. The same axisymmetric Poisson equation also applies either in the presence of an axisymmetric beam pipe or a nonaxisymmetric pipe provided that the pipe is sufficiently far outside the characteristic radial extent of the beam where the boundary conditions have negligible influence on the beam. We henceforth assume that either of these conditions apply.

\section{B. Single-species plasma confined in a Penning-Malmberg Trap}

Although the model described here is typically applied to a pure electron plasma confined in a Penning-Malmberg trap confinement geometry, ${ }^{1,4}$ for generality, we do not assume a particular species of particle. Real non-neutral plasma systems have finite axial length and longitudinal confinement is typically provided by an applied axial potential well. The two-dimensional plasma model described can be annlied to model the middle portion of an axially long plasma column where end effects can be neglected, or to model a shorter plasma when the axial bounce motion of the particles is fast relative to characteristic transverse evolutions in a sense that enables interpretation of the equations in an axial bounce-averaged sense.

Assume a pure non-neutral plasma composed of particles of charge $q$ and mass $m$ confined radially by a uniform axial magnetic field $B \hat{\mathbf{z}}=$ const. The nonrelativistic Hamiltonian $H$ and canonical angular momentum $P_{\theta}$ of a singleparticle are given by

$$
\begin{aligned}
& H=\frac{\mathbf{p}^{2}}{2 m}+q \phi, \\
& P_{\theta}=x p_{y}-y p_{x}+\frac{m \omega_{c}}{2} r^{2} .
\end{aligned}
$$

Here, $\mathbf{x}=x \hat{\mathbf{x}}+y \hat{\mathbf{y}}+z \hat{\mathbf{z}}$ and $\mathbf{p}=p_{x} \hat{\mathbf{x}}+p_{y} \hat{\mathbf{y}}+p_{z} \hat{\mathbf{z}}$ are the threedimensional particle coordinates and mechanical momentum, $r=\sqrt{x^{2}+y^{2}}$ is the radial cylindrical coordinate, $\phi$ is the electrostatic potential, and $\omega_{c}=q B /(m c)$ is the cyclotron frequency. Thermal equilibrium is specified by the special form of three-dimensional rigid-rotor distribution given by ${ }^{1}$

$$
f(\mathbf{x}, \mathbf{p})=\frac{\hat{n}}{(2 \pi m T)^{3 / 2}} \exp \left[-\frac{\left(H+\omega_{r} P_{\theta}\right)}{T}\right] .
$$

Here, $T=$ const is the thermodynamic temperature (energy units), $\hat{n}=$ const is a characteristic density scale, and $\omega_{r}=$ const is an angular velocity. For the choice of distribution in Eq. (6), $\omega_{r}$ is identified as the frequency of rigid rotation of the plasma column, i.e., the azimuthal plasma flow velocity along $\hat{\theta}=-\sin \theta \hat{\mathbf{x}}+\cos \theta \hat{\mathbf{y}}$ is

$$
V_{\theta}=\frac{\int d^{3} p \frac{\mathbf{p} \cdot \hat{\theta}}{m} f}{\int d^{3} p f}=-\omega_{r} r .
$$

The plasma density is directly calculable from $\phi$ as

$$
n=\int d^{3} p f=\hat{n} \exp \left\{-\frac{m}{2 T}\left[\left(\omega_{r} \omega_{c}-\omega_{r}^{2}\right) r^{2}+\frac{2 q}{m} \phi\right]\right\} .
$$

Assuming an equilibrium with $\partial / \partial z=0$, the proof in Ref. 21 can be applied to show that the solution to Poisson's equation in radially unbounded geometry is axisymmetric $(\partial / \partial \theta=0)$, and the three-dimensional Poisson equation $\nabla^{2} \phi=-4 \pi q n$ reduces to

$$
\frac{1}{r} \frac{\partial}{\partial r} r \frac{\partial}{\partial r} \phi=-4 \pi q \hat{n} \exp \left\{-\frac{m}{2 T}\left[\left(\omega_{r} \omega_{c}-\omega_{r}^{2}\right) r^{2}+\frac{2 q}{m} \phi\right]\right\} \text {. }
$$

Without loss of generality, we take a potential reference choice of $\phi(r=0)=0$ and consistently identify the on-axis density of the plasma column as $n(r=0)=\hat{n}$. It can be shown that the plasma column is radially confined provided that ${ }^{1}$

$$
\omega_{c} \omega_{r}-\omega_{r}^{2}-\frac{1}{2} \omega_{p}^{2}>0,
$$

where $\omega_{p}=\sqrt{4 \pi q^{2} \hat{n} / m}$ is the plasma frequency. If this condition is met, the plasma is radially bounded and $n(r)$ is a 
TABLE I. Scaled variables in the transformed Poisson equation. In both formulations, $\omega_{p}=\sqrt{4 \pi q^{2} \hat{n} / m}$ and $\lambda_{D}=\sqrt{T /\left(m \omega_{p}^{2}\right)}$ are the nonrelativistic plasma frequency and Debye length formed from the on-axis density $n(r=0)=\hat{n}$.

\begin{tabular}{lcc}
\hline \hline Variable & Continuous focusing beam & Penning-Malmberg trap \\
\hline$\rho=$ & $\frac{r}{\gamma_{b} \lambda_{D}}$ & $\frac{r}{\lambda_{D}}$ \\
$\psi=$ & $\frac{m \gamma_{b} \beta_{b}^{2} c^{2} k_{\beta 0}^{2}}{2 T} r^{2}+\frac{q}{\gamma_{b}^{2} T} \phi$ & $\frac{m}{2 T}\left(\omega_{r} \omega_{c}-\omega_{r}^{2}\right) r^{2}+\frac{q}{T} \phi$ \\
$\Delta=$ & $\frac{2 \gamma_{b}^{3} \beta_{b}^{2} c^{2} k_{\beta 0}^{2}}{\omega_{p}^{2}}-1$ & $\frac{2\left(\omega_{r} \omega_{c}-\omega_{r}^{2}\right)}{\omega_{p}^{2}}-1$ \\
\hline \hline
\end{tabular}

monotonically decreasing function of $r$ with $\partial n / \partial r<0$. Similar to the beam case in Sec. II A, the Poisson equation can also be applied to a plasma in a conducting pipe provided that the pipe is sufficiently outside the radial extent of the plasma.

\section{Scaled equations describing the thermal equilibrium potential or density profile}

For either the beam or plasma models described in Secs. II A and II B, the equilibrium Poisson Eqs. (4) or (9) can be expressed in scaled form as ${ }^{1,3,4}$

$$
\frac{1}{\rho} \frac{\partial}{\partial \rho}\left(\rho \frac{\partial \psi}{\partial \rho}\right)=1+\Delta-e^{-\psi},
$$

and is solved subject to $\psi(\rho=0)=0=[\partial \psi / \partial \rho]_{\rho=0}$. Here, $\psi$ is a dimensionless effective potential, $\rho$ is a dimensionless radial coordinate measured in terms of characteristic Debye lengths, and $\Delta \in(\infty, 0)$ is a dimensionless parameter that measures the ratio of applied focusing to space-charge defocusing strength. Definitions of these variables for both the beam and plasma models are given in Table I. Values of $\Delta \gtrsim 1$ correspond to a warm equilibrium with space-charge forces less than the applied focusing forces. Conversely, values of $\Delta \ll 1$ correspond to high space-charge intensity with space-charge forces nearly canceling the linear applied focusing strength within the core of the equilibrium. Very small values of $\Delta$ with $\Delta \leq 10^{-6}$ are common in a variety of beam and non-neutral plasma experiments with strong relative space-charge.

The Poisson equation (11) can be alternatively expressed in terms of the normalized density

$$
N \equiv \frac{n}{\hat{n}}=e^{-\psi}
$$

as $^{16,17}$

$$
\frac{\partial^{2} N}{\partial \rho^{2}}-\frac{1}{N}\left(\frac{\partial N}{\partial \rho}\right)^{2}+\frac{1}{\rho} \frac{\partial N}{\partial \rho}=N^{2}-(1+\Delta) N
$$

with $N(\rho=0)=1$ and $[\partial N / \partial \rho]_{\rho=0}=0$. For thermal equilibrium, the scaled potential $\psi$ can be simply calculated in terms of scaled density $N$ from Eq. (12) as $\psi=-\ln N$. Because the structure of the equilibrium is easier to visualize in terms of

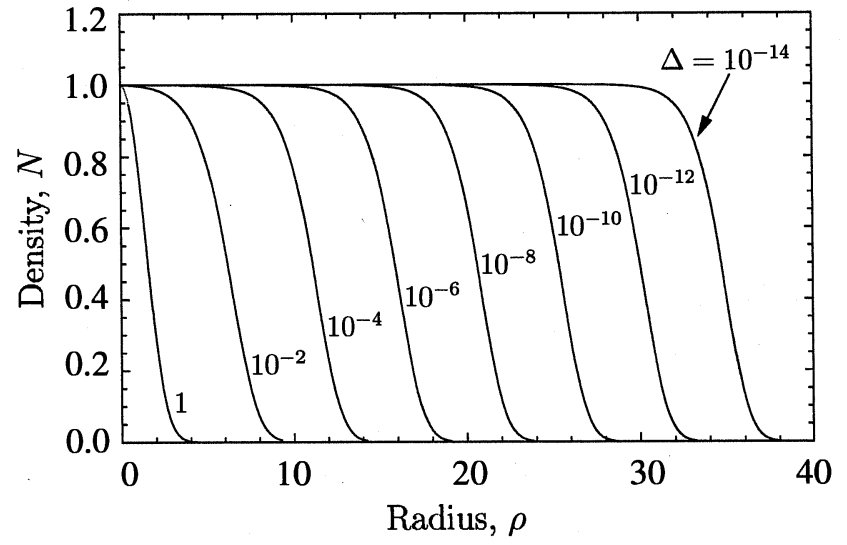

FIG. 1. Numerical calculation of scaled density $N$ vs scaled radial coordinate $\rho$ for the values of $\Delta$ indicated.

$N$ rather than $\psi$, we present equilibrium solutions primarily in terms of $N$ in the following sections.

\section{Properties of thermal equilibrium}

To illustrate the thermal equilibrium distribution, numerical solutions of Eq. (13) for the normalized density $N$ are presented in Fig. 1 for a wide range of $\Delta$ values. For $\Delta \sim 1$ and larger, the solution appears Gaussian-like. Conversely, for $\Delta \ll 1, N$ varies little from unity from $\rho=0$ until $\rho$ is sufficiently large number of Debye lengths where $N$ rapidly falls to exponentially small values over a range of 4-5 Debye lengths. In this regime, note that the radial width and structure of the edge in $\rho$ varies little with $\Delta$, whereas the width of the flat central region where $N \simeq 1$ is a strong function of $\Delta$. Strong Debye screening ${ }^{1-4}$ results in a flat core as space-charge screens the linear applied focusing forces. The finite amount of equilibrium charge results in a density profile edge with the same characteristic radial falloff regardless of the radial extent of the core. The core of the density profile becomes exceedingly flat for very small values of $\Delta$ (i.e., at $\rho=0 ; N=1, \partial N / \partial \rho=0$, and $\partial^{2} N / \partial \rho^{2}=-\Delta / 2$ ). This extreme flatness can lead to difficulties when integrating Eq. (11) and/or Eq. (13) from $\rho=0$ using standard, fixed precision numerical methods. Early papers do not appear to address this difficulty and avoid parameters with $\Delta \leq 10^{-6}$ to allow use of standard numerical methods. ${ }^{1-4,11-15}$ However, realizable laboratory systems can easily correspond to much lower values of $\Delta$. A numerical method has been derived (see Appendices F and G of Ref. 17) to accurately calculate the solution for arbitrarily small values of $\Delta>0$. This method employs a nonlinear power series solution ${ }^{16,17}$ that converges out to a cutoff value of $\rho$ where the density profile edge in $N$ is slightly less than unity to analytically describe the core. Then the analytical core solution is extended outward from the cutoff radius in $\rho$ using a numerical solution to the equilibrium equation. ${ }^{17}$ In this region of rapid variation the series solution fails to converge, but the numerical solution can be constructed using conventional numerical methods for the solution of ordinary differential equations. 
Constraint equations are derived and analyzed in Appendix F of Ref. 17 that relate $\Delta$ and other choices of scaled variables describing the thermal equilibrium to values of the thermal temperature $T$ as well as standard choices of accelerator parameters such as the focusing strength, perveance, rms edge radius, and rms edge emittance. Generally, ranges of physical beam parameters can correspond to a single value of $\Delta$ and the same normalized density profile. However, the value of $\Delta$ uniquely specifies the relative strength of spacecharge to applied focusing forces in the thermal equilibrium beam. Analogous parameter constraints can be derived for an unneutralized, single-species plasma in a Penning-Malmberg trap confinement geometry in terms of convenient variables. ${ }^{4}$ Parameter eliminations will typically require solution of highly nonlinear constraint equations as illustrated in Ref. 17. Other properties of thermal equilibrium can be calculated in terms of $\Delta, N$, and the choice of the physical system scale as illustrated in Ref. 17.

\section{APPROXIMATE ANALYTICAL SOLUTIONS DESCRIBING THE THERMAL EQUILIBRIUM DENSITY PROFILE}

In the regime of weak space-charge forces relative to the applied focusing forces, $\Delta \gg 1$. If $\Delta$ is sufficiently large such that $1+\Delta \gg e^{-\psi}$, then the transformed equilibrium Poisson equation (11) can be solved analytically as

$$
\psi \simeq \frac{1+\Delta}{4} \rho^{2}
$$

and the scaled density $N=e^{-\psi}$ [see Eq. (12)] has Gaussian form with

$$
N \simeq \exp \left[-\frac{1+\Delta}{4} \rho^{2}\right] .
$$

The Gaussian radial density profile is expected for a thermal distribution subject to linear focusing forces (see Fig. 1). Denote the numerical solution to Eq. (13) for $N$ as $N_{\text {num }}$ and the approximate, large- $\Delta$ Gaussian-form solution for $N$ given by Eq. (15) as $N_{\text {app }}$. In Fig. 2(a), the fractional error $\left|N_{\text {app }}-N_{\text {num }}\right| / N_{\text {num }}$ is plotted on a logarithmic scale as a function of $\rho$ for several values of $\Delta$. Curves are cutoff where $N$ becomes small. The approximate solution $N_{\text {app }}$ is accurate over a wide range of $\rho$ when $\Delta>0.1$. The largest fractional errors are characteristically in the low density tail of $N$ because weak space-charge acts to sharpen the radial profile of $N$. These errors will not be significant for many applications.

In the regime of strong relative space-charge strength, $\Delta \ll 1$, and Eqs. (11) and/or (13) are highly nonlinear and have previously been analyzed numerically ${ }^{1-4,11-15}$ or with a nonlinear series expansion valid only within the core of $N{ }^{16,17}$ Here we derive an approximate, closed-form analytical solution for $N$. For $\Delta \ll 1$, the solution $N$ to Eq. (11) is almost unity everywhere except over a several Debye-length radial distance from the edge of the density profile, where $N$ falls smoothly to zero. Inside the core, where $N \simeq 1$, we approximate $N=1+\Delta-\eta$ with $|\eta| \ll 1$. In this region, the function $\eta(\rho)$ satisfies the linearized version of Eq. (13),
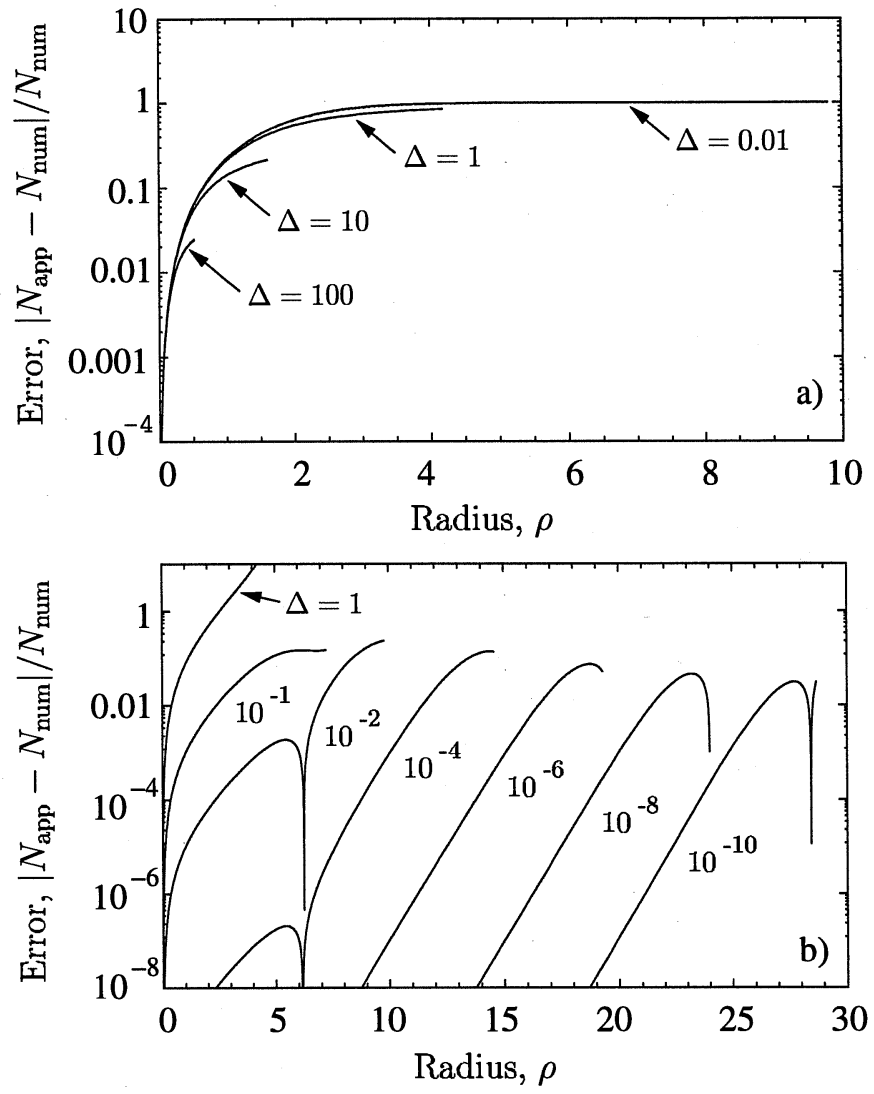

FIG. 2. Fractional errors of approximate solution $\left|N_{\text {app }}-N_{\text {num }}\right| / N_{\text {num }}$ are plotted as a function of $\rho$ for the indicated values of $\Delta$ for (a) weak spacecharge (large $\Delta$ ), and (b) strong space-charge (small $\Delta$ ). Curves are cutoff at values of $\rho$ where $N_{\text {num }}(\rho)=0.001$.

$$
\frac{\partial^{2} \eta}{\partial \rho^{2}}+\frac{1}{\rho} \frac{\partial \eta}{\partial \rho}-\eta=0
$$

subject to the boundary conditions $\eta(\rho=0)=\Delta$ and $\partial \eta /\left.\partial \rho\right|_{\rho=0}=0$. Here we have taken $(1+\Delta) \eta \simeq \eta$. Equation (16) is recognized as the equation of a modified Bessel function of order zero. ${ }^{22}$ The solution is regular at $\rho=0$ satisfying the boundary condition is $\eta(\rho)=\Delta I_{0}(\rho)$, where $I_{\nu}(x)$ denotes an order $\nu$ modified Bessel function.

The approximate solution $N=1+\Delta\left[1-I_{0}(\rho)\right]$ is applicable everywhere where $\Delta I_{0}(\rho) \ll 1$. In the same region, $\psi=-\ln N \simeq-\Delta\left[1-I_{0}(\rho)\right]$. We search for a solution to Eq. (11) valid in the region where $\Delta I_{0}(\rho) \sim 1$ by taking $\psi=F(\eta)-\Delta$ with $\eta \equiv \Delta I_{0}(\rho)$. Substituting this ansatz into Eq. (11) and employing Eq. (16) to simplify the resulting expression, we obtain the differential equation for the function $F(\eta)$,

$$
\begin{aligned}
\eta F_{\eta}+\left[\Delta \frac{\partial I_{0}(\rho)}{\partial \rho}\right]^{2} F_{\eta \eta} & =1+\Delta-\exp (\Delta-F) \\
& \simeq(1+\Delta)[1-\exp (-F)] .
\end{aligned}
$$

Here and henceforth, subscripts are employed to denote derivatives with respect to the argument, e.g., $F_{\eta} \equiv \partial F(\eta) / \partial \eta$. In the region where $\eta=\Delta I_{0}(\rho) \gg \Delta$, we approximate $\partial I_{0}(\rho) / \partial \rho=I_{1}(\rho) \simeq I_{0}(\rho)$ when $\Delta \ll 1$. This introduces an error $\delta \psi \sim 1 / \ln (1 / \Delta)$. With this approximation, Eq. (17) reduces to 


$$
F_{\xi \xi}=1-\exp (-F),
$$

where $\quad \xi=[1+\Delta]^{1 / 2} \ln (\eta)=[1+\Delta]^{1 / 2} \ln \left[\Delta I_{0}(\rho)\right] \simeq \ln \left[\Delta I_{0}(\rho)\right]$. Therefore, we are looking for a solution of Eq. (18) asymptotically approaching $F(\xi)=\exp (\xi)$ for $\ln (\Delta) \ll \xi \ll-1$. Integrating Eq. (18) once, we obtain

$$
\frac{\left(F_{\xi}\right)^{2}}{2}=F+\exp (-F)-1 \text {, }
$$

where the constant of integration is chosen to obtain the correct asymptotic behavior $F(\xi)=\exp (\xi) \ll 1$ for $\ln (\Delta) \ll \xi \ll-1$.

The solution of Eq. (19) satisfying the limiting form $F(\eta)=\eta$ for $\eta \ll 1$ can be expressed in integral form as

$$
\int_{\epsilon}^{F} \frac{d f}{\sqrt{\exp (-f)+f-1}}=\sqrt{2}(\xi-\ln \epsilon),
$$

where $\epsilon \rightarrow 0^{+}$. The integral in Eq. (20) is not simply expressible in terms of elementary functions. To obtain a simplified approximation for the solution, we first make the change of variables $F=2 \ln G$. The new variable $G$ is related to density $N$ by $N=\exp (\Delta) / G^{2}$. In terms of $G$, Eq. (20) becomes

$$
\int_{\exp (\epsilon / 2)}^{G} \frac{d g}{\sqrt{1-g^{2}+g^{2} \ln \left(g^{2}\right)}}=\frac{1}{\sqrt{2}}(\xi-\ln \epsilon) .
$$

We now approximate the expression under the root in Eq. (21) with its Taylor expansion about $g=1$, by taking $1-g^{2}+g^{2} \ln \left(g^{2}\right) \simeq 2(g-1)^{2}+\frac{2}{3}(g-1)^{3}$. This approximation introduces an error in the final result for the density $N$ of order $\delta N \sim 1 / \ln (1 / \Delta)$. After this substitution, we integrate, take the limit $\epsilon \rightarrow 0^{+}$, and obtain

$$
G=1+\frac{\exp (\xi)}{2[1-\exp (\xi) / 24]^{2}},
$$

or in terms of density $N=\exp (\Delta) / G^{2} \simeq(1+\Delta) / G^{2}$,

$$
N \simeq \frac{1+\Delta}{\left\{1+\frac{\Delta I_{0}(\rho)}{2} /\left[1-\frac{\Delta I_{0}(\rho)}{24}\right]^{2}\right\}^{2}} .
$$

From Eq. (23), we see that this solution is only applicable where $\Delta I_{0}(\rho) / 24 \ll 1$. In this region, we can further simplify Eq. (23) as

$$
N \simeq \frac{\left(1+\frac{1}{2} \Delta+\frac{1}{24} \Delta^{2}\right)^{2}}{\left\{1+\frac{1}{2} \Delta I_{0}(\rho)+\frac{1}{24}\left[\Delta I_{0}(\rho)\right]^{2}\right\}^{2}} .
$$

Here we have inserted a small correction terms $\propto \Delta^{2}$ and higher-order in $\Delta$ in the numerator to enforce $N(\rho=0)=1$ exactly - regardless of the size of $\Delta$. The value of density below which the approximate Eq. (24) becomes invalid is found by substituting $\Delta I_{0}(\rho) / 24=1$ into Eq. (24) and is found to be approximately $N \simeq 0.001$.

Equation (24) gives our final, approximate solution to Eq. (13) for the density $N$ over the entire range of $\rho$. This solution is expected to be valid for $\Delta \ll 1$. Denote the approximate, small- $\Delta$ solution in Eq. (24) as $N_{\text {app. }}$ In Fig. 2(b), the fractional error $\left|N_{\text {app }}-N_{\text {num }}\right| / N_{\text {num }}$ is plotted on a loga-

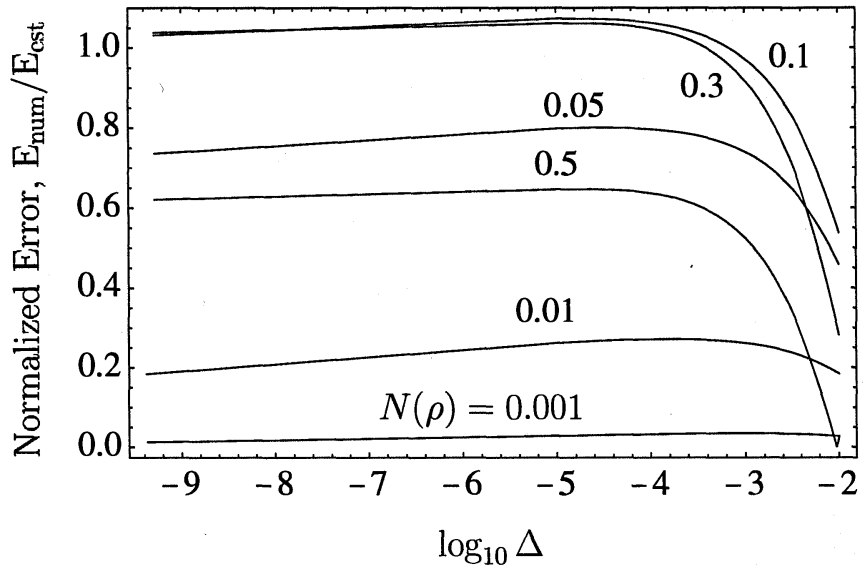

FIG. 3. Normalized error $E_{\text {num }} / E_{\text {est }}$ plotted as a function of $\log _{10} \Delta$ for values of $\rho$ satisfying $N_{\text {num }}(\rho)=0.001,0.01,0.05,0.1,0.3$, and 0.5 as indicated on the curves. Here, $E_{\text {num }}=\left|N_{\text {app }}-N_{\text {num }}\right| / N_{\text {num }}$ and $E_{\text {est }}=[0.04 / \ln (1 / \Delta)] / N_{\text {num }}$.

rithmic scale as a function of $\rho$ for several values of $\Delta$. The manner of presentation is the same as in Fig. 2(a) for the large- $\Delta$ solution. Errors in the central core are extremely small-plots are cutoff at $10^{-8}$ to avoid uncertainties associated with the finite accuracy of the numerical methods employed. The approximate solution $N_{\text {app }}$ is accurate over a wide range of $\rho$ when $\Delta<0.1$ with the highest errors in the far tail. The total error introduced by the approximation scales as $\delta N \simeq \lambda / \ln (1 / \Delta)$, where the coefficient $\lambda=$ const is estimated numerically as $\lambda \sim 0.04$. To verify this error scaling, Fig. 3 shows the fractional error $E_{\text {num }}(\rho) \equiv \mid N_{\text {app }}(\rho)$ $-N_{\text {num }}(\rho) \mid / N_{\text {num }}(\rho)$ normalized to the error estimate $E_{\text {est }}(\rho)$ $\equiv[0.04 / \ln (1 / \Delta)] / N_{\text {num }}(\rho)$ plotted as a function of $\log _{10} \Delta$. This normalized error is a function of $\rho$ and curves are shown for $\rho=\rho(\Delta)$, where $\rho$ satisfies $N_{\text {num }}(\rho)=$ const with the constant corresponding to the indicated values within the radial edge of the density profile.

To better understand the errors in the tenuous tail of $N$, we proceed as follows. Denote the value of $\rho$ where $N=1 / 2$ by $\rho=\rho_{1 / 2}$. If we approximate $\left(1+\frac{1}{2} \Delta+\frac{1}{24} \Delta^{2}\right)^{2} \simeq 1$ in Eq. (24), one finds that $\rho_{1 / 2}$ satisfies the constraint equation $\Delta I_{0}\left(\rho_{1 / 2}\right)=C$, where $C=2 \sqrt{3+6 \sqrt{2}}-6 \simeq 0.77799$. If $\Delta \ll 1$, and consistently, $\rho_{1 / 2} \gg 1$, then the asymptotic formula ${ }^{22}$ $I_{0}(x) \simeq(1 / \sqrt{2 \pi x}) e^{x}$ valid for $x \gg 1$, can be applied to derive a simple, approximate expression for $\rho_{1 / 2}$ :

$$
\rho_{1 / 2} \simeq \ln \left[\frac{C}{\Delta} \sqrt{2 \pi \ln \left(\frac{C}{\Delta}\right)}\right] .
$$

In the region radially well outside the core of the density profile, $\rho \gg \rho_{1 / 2}$ and $N \ll 1$. In this region, $1+\Delta \gg e^{-\psi}$ and Eq. (15) shows that $N \sim \exp \left[-(1+\Delta) \rho^{2} / 4\right]$, whereas the approximate solution in Eq. (23) varies as $N \sim \exp (-4 \rho)$. Thus, the approximate solution is poor within the tenuous, lowdensity tails of the equilibrium while accurately modeling the core and radial falloff of the edge. This deficiency will not matter for many applications. 


\section{CONCLUSIONS}

A new approximate analytical solution has been derived that accurately models the radial density or potential profile of a thermal equilibrium distribution describing either a beam in a continuous focusing channel or a single-species non-neutral plasma confined in a Penning-Malmberg trap. The scaled solution is a function of a radial coordinate expressed in terms of Debye lengths and depends only on a single dimensionless parameter $\Delta$. The parameter $\Delta \in(\infty, 0)$ measures the strength of space-charge defocusing forces relative to linear applied focusing forces acting on the equilibrium. The solution is highly accurate for $\Delta<0.1$, which corresponds to a broad range of strong relative spacecharge strength and produces a highly nonlinear density profile with a flat core region characteristic of strong Debye screening. The solution can be employed in a variety of practical problems in beam and non-neutral plasma physics. For example, the solution could be applied to calculate simplified expressions for the distribution of particle frequencies and angular momentum in thermal equilibrium with strong space-charge.

\section{ACKNOWLEDGMENTS}

The authors wish to thank R. C. Davidson for useful discussions. This research was performed under the auspices of the U.S. Department of Energy at Princeton Plasma Physics Laboratory under Contract No. DE-ACOZ-76-CHO-3073 and Lawrence Livermore National Laboratory under Contract No. DE-AC52-07NA27344.
${ }^{1}$ R. C. Davidson, Physics of Non-Neutral Plasmas (Addison-Wesley, Reading, 1990; rereleased World Scientific, New York, 2001), and references therein.

${ }^{2}$ M. Reiser, Theory and Design of Charged Particle Beams (Wiley, New York, 1994), and references therein.

${ }^{3}$ R. C. Davidson and H. Qin, Physics of Intense Charged Particle Beams in High Energy Accelerators (World Scientific, New York, 2001), and references therein.

${ }^{4}$ D. H. E. Dubin and T. M. O'Neil, Rev. Mod. Phys. 71, 87 (1999), and references therein.

${ }^{5}$ T. K. Fowler, J. Math. Phys. 4, 559 (1963).

${ }^{6}$ C. S. Gardner, Phys. Fluids 6, 839 (1963).

${ }^{7}$ R. C. Davidson, Phys. Rev. Lett. 81, 991 (1998).

${ }^{8}$ R. C. Davidson, J. Plasma Phys. 6, 229 (1971).

${ }^{9} \mathrm{M}$. Reiser, Theory and Design of Charged Particle Beams (Wiley, New York, 1994).

${ }^{10}$ E. A. Startsev and R. C. Davidson, Phys. Rev. ST Accel. Beams 6, 044401 (2003).

${ }^{11}$ J. D. Lawson, The Physics of Charged-Particle Beams (Clarendon, Oxford, 1977).

${ }^{12}$ T. M. O'Neil and C. F. Driscoll, Phys. Fluids 22, 266 (1979).

${ }^{13}$ S. A. Prasad and T. M. O'Neil, Phys. Fluids 22, 278 (1979).

${ }^{14}$ M. Reiser and N. Brown, Phys. Rev. Lett. 71, 2914 (1993).

${ }^{15}$ R. C. Davidson and H. Qin, Phys. Rev. ST Accel. Beams 2, 114401 (1999).

${ }^{16}$ R. C. Davidson and S. M. Lund, AIP Conf. Proc. 314, 1 (1994).

${ }^{17}$ S. M. Lund, T. Kikuchi, and R. C. Davidson, "Generation of initial kinetic distributions for simulation of long-pule charged particle beams with high space-charge intensity," Phys. Rev. ST Accel. Beams (submitted).

${ }^{18}$ S. M. Lund and B. Bukh, Phys. Rev. ST Accel. Beams 7, 024801 (2004).

${ }^{19} \mathrm{~S}$. M. Lund and S. R. Chawla, Nucl. Instrum. Methods Phys. Res. A 561, 203 (2006).

${ }^{20}$ S. M. Lund, J. J. Barnard, B. Bukh, S. R. Chawla, and S. H. Chilton, Nucl. Instrum. Methods Phys. Res. A 577, 173 (2007).

${ }^{21}$ S. M. Lund, Phys. Rev. ST Accel. Beams 10, 064203 (2007).

${ }^{22}$ Handbook of Mathematical Functions with Formulas, Graphs, and Mathematical Tables, 9th ed., edited by M. Abramowitz and I. A. Stegun (Dover, New York, 1972). 